\title{
Characterization of transmission line based on advanced SOLTcalibration: Review
}

\author{
${ }^{1}$ Abhishek Agrawal, ${ }^{2}$ Alpana Pandey, ${ }^{3}$ Vikas Dhanda \\ ${ }^{I}$ Department of Electronics and CommunicationM.A.N.I.T. Bhopal \\ ${ }^{2}$ Department of Electronics and CommunicationM.A.N.I.T. Bhopal \\ ${ }^{3}$ Design Engineer STMicroelectronics Pvt. Ltd.
}

\begin{abstract}
Validation of analog IP's at high frequency is performed on a test board. For proper validation of IP's, it is very necessary to characterize the transmission line in such a manner that how much amount of signal is received at the destination \& how much amount of losses introduce the transmission line. This paper proposed, Fixture free characterization of transmission line mounted on test board, based on advanced SOLT calibration performed on test dummy structure (ISS). Extraction of RLGC parameters, attenuation constant and phase constant of transmission line in very simple manner. Advanced probes technology minimizes insertion and return losses at high frequency which introduce by the adopters. Fixture free characterization minimizes phase delays produced by the adopters.
\end{abstract}

Keywords: thetransmission line, SOLT Calibration, Test dummy structure (ISS), ACP GS probes

\section{Introduction}

Fixture at RF frequency introduces insertion \& return loss. As frequency increases attenuation losses are also increases which contribute inaccuracy in the measurement of S parameter [1]. Earlier there are different methods discovered for characterization of DUT. In order to meet these high speed requirements the design engineer must consider and minimize all the factors that impact the integrity of the digital signals in order to ensure the correct system functioning [7]. Adapting a coaxial test cable to a Microstrip device requires an adequate test fixture and a suitable de-embedding technique for removing the effects of the test fixture from the device under test (DUT). This further evaluates the de-embedding problem and the phase errors introduced when using test fixtures [7]. At RF improved accuracy \& reduced attenuation losses achieved by fixture free measurement of DUT \& well calibrates probes using SOLT calibration performed on wafer ISS (Short, open, load, thru).

\section{A. Port Extension Method}

\section{Methods used FOR DUT characterization}

It is used to remove the effects of the test fixture. It shifts the reference plane till the ends of the fixture but it is less accurate in practical environments. As the number of the fixtures increases inaccuracy in measurement is also increased. Advancement of port extension is the adopter removal method.

\section{B. Adopter Removal Method}

According to [1], there are generally two types of approaches to remove the effects of test fixtures.

The first approach uses specialized calibration standards that are inserted at the end of the test fixture, and performing a calibration process to move the reference plane to the end of the test fixture. The accuracy of the measurement relies highly on the accuracy of the physical calibration standards. It is not practical to build an accurate broadband SOLT (short-open-load-thru) right after the test fixture; hence the traditional SOLT calibration process is generally not applicable for test fixture de-embedding. Alternatively, the TRL (thrureflect-line) and LRM (line-reflect-match) method is a more suitable approach.

The second approach makes direct S parametermeasurementsof the DUT with test fixture, meanwhile acquires the S parameter of the test fixture through either direct measurement or simulation. The $\mathrm{S}$ parameter of the DUT without test fixture can be mathematically calculated from above two $\mathrm{S}$ parameter data.

According to [2], the second approach with S parameter de-embedding yields the best results to the ideal DUT response. It is gaining popularity due to its straightforwardness in measurement, and easiness of data post processing. 


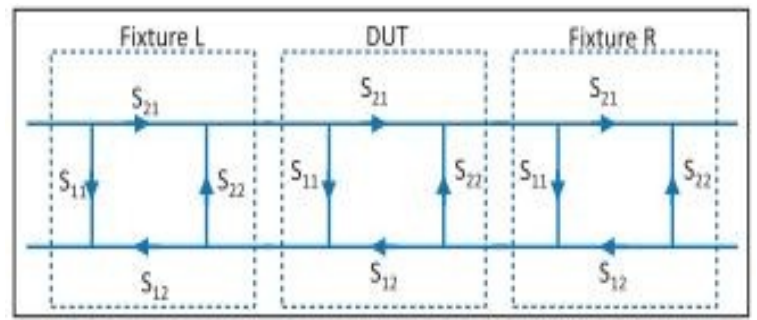

Figure 1: Signal flow chart of the $\mathrm{S}$ parameters of test fixtures and DUT

When $\left[T_{L}\right] \&\left[T_{R}\right]$ are known then de-embedding result is:

$\left[\mathrm{T}_{\text {De-embedded }}\right]=\left[\mathrm{T}_{\mathrm{R}}^{-1}\right]\left[\mathrm{T}_{\text {DUT }}\right]\left[\mathrm{T}_{\mathrm{L}}^{-1}\right]$

$$
=\left[\mathrm{T}_{\mathrm{R}}^{-1}\right]\left[\mathrm{T}_{\mathrm{R}}\right]\left[\mathrm{T}_{\mathrm{DUT}}\right]\left[\mathrm{T}_{\mathrm{L}}\right]\left[\mathrm{T}_{\mathrm{L}}^{-1}\right]
$$

$$
=\left[\mathrm{T}_{\mathrm{DUT}}\right]
$$

In practical environment Fixture like SMA is connected to the transmission line (DUT) on test board using solders .With the help of this method we removes the effect of SMA but solders' effect is still present. This solder cause impedance mismatch at the board end \& creates in-accuracy at higher frequency. Due to impedance mismatch at the board end S parameter of de-embedded fixture is slightly different. This amplifies the error by an amount of $\frac{\text { S22.S11 }}{\text { S21.S21 }}$.

\section{De-embedding Technique For Phase Delay Associated by Connectors}

According to [7] due to fixture, phase delay is introduced into the measurement. The fixture Sparameter model can be simplified assuming a Microstrip with matched characteristic impedance of 50 2 thus the reflection coefficients (S11 and S22) at M1 and M2 are reduced to zero. S21 and S12of the Microstrip can be defined in terms of quasi-TEM wave equations with complex propagation constant and length $\mathrm{L}$.

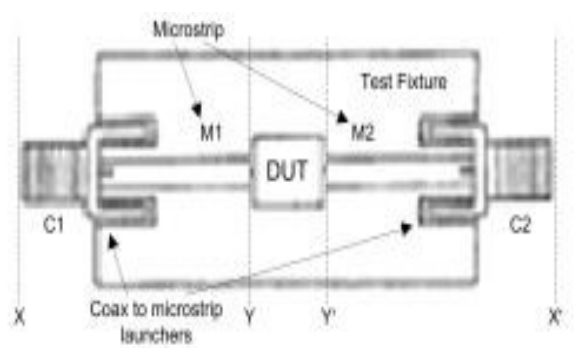

Figure 2: Test fixture

$$
[S]=\left[\begin{array}{cc}
\mathrm{S} 11 \mathrm{e}^{-\gamma 11} & \mathrm{~S} 12 \mathrm{e}^{-\gamma(11+12)} \\
\mathrm{S} 21 \mathrm{e}^{-\gamma(11+12)} & \mathrm{S} 22 \mathrm{e}^{-\gamma 12}
\end{array}\right]
$$

However, when building an actual test fixture, any change $\Delta$ lin the connector geometry or Microstripwill introduce error into the final DUT S-parameters. Suppose we concentrate specifically on the effect of a length deviation in the Microstrip. In matrix form the S-parameter error introduced by changing the Microstrip lengths is given by

$$
[\mathrm{S}]=\left[\begin{array}{cc}
\mathrm{S} 11 \mathrm{e}^{-\gamma(2 . \Delta 11)} & \mathrm{S} 12 \mathrm{e}^{-\gamma(2 . \Delta 11+12)} \\
\mathrm{S} 21 \mathrm{e}^{-\gamma(2 . \Delta 11+12)} & \mathrm{S} 22 \mathrm{e}^{-\gamma(2 . \Delta 12)}
\end{array}\right]
$$

The phase error (in radians) for each S-parameter of Microstrip is:

$$
[\Delta \varphi \mathrm{f}]=\left[\begin{array}{cc}
\frac{4 \pi \mathrm{f} \Delta \mathrm{l} 1 \sqrt{\varepsilon e f f}}{\mathrm{c}} & \frac{2 \pi \mathrm{f}(\Delta \mathrm{ll} 1+\Delta \mathrm{l} 2) \sqrt{\varepsilon \mathrm{eff}}}{\mathrm{c}} \\
\frac{2 \pi \mathrm{f}(\Delta \mathrm{l} 1+\Delta \mathrm{l} 2) \sqrt{\varepsilon e f f}}{\mathrm{c}} & \frac{4 \pi \mathrm{f} \Delta \mathrm{l} 2 \sqrt{\varepsilon \mathrm{eff}}}{\mathrm{c}}
\end{array}\right]
$$

\section{New De-Embedding Method Based On Solt Calibration On Impedance Substrate Structure}

According to [5], all losses due to impedance mismatching or due to fixtures are removed using fixture free measurement of DUT, based on advanced SOLT calibration performed on the ISS \& advanced ACP GSG 
probes. By using mechanical calibration it is impossible to shift the reference plane till the ends of fixture. This calibration shifts the reference plane till the ends of probes' tip. It uses 2 ISS. One ISS have open, short \& load structure whereasaccuracy in measurement of $\mathrm{S}$ parameter is depends on this calibration. This de-embedding method overcomes the drawback of the other Known solutions. Propagation constant \& characteristic impedance of transmission line can be defined by eq. (1) \& (3)

$\gamma=\sqrt{(R+j \omega L)(G+j \omega C)}(1)$

$\gamma=\alpha+\mathrm{j} \beta(2)$

$\mathrm{Zo}=\frac{\sqrt{\mathrm{R}+\mathrm{j} \omega \mathrm{L}}}{\sqrt{\mathrm{G}+\mathrm{j} \omega \mathrm{C}}}(3)$

By solving these three equations we got

$\mathrm{G}=\frac{\alpha}{\mathrm{Zo}}(4)$

$\mathrm{C}=\frac{\beta}{\omega \mathrm{Zo}}(5)$

$\mathrm{R}=\mathrm{Zo}^{2} \mathrm{G}(6)$

$\mathrm{L}=\mathrm{Zo}^{2} \mathrm{C}(7)$

$\mathrm{R}$ is loop resistance per unit length, represents the lossy behavior of conductor. $\mathrm{G}$ is shunt conductance per unit length, represents lossy behavior of dielectric. L is the loop inductance per unit length,which store magnetic energy. $\mathrm{C}$ is shunt capacitance per unit length, represents the electric energy.

\section{Measurement Setup}

On the basis of previous work a measurement is performed on $5 \mathrm{~cm}$ long single ended transmission line can be used for validation of this method. Transmission line is mounted on PCB \& use FR4 dielectric material whose permittivity is 4.8. First step is performing 2 port measurement of transmission line of test board for extraction of other parameters. This measurement performed using 4 ports Agilent PNA X N5244A Network Analyzer having a broad frequency range of $10 \mathrm{MHz}$ to $43.5 \mathrm{GHz}$. BTS 2000 used to holding the probes. High bandwidth coaxial cable $(40 \mathrm{GHz})$ is connected to one end of PNA ports \& another side of coaxial cable is connected to DUT via ACP probe. Single ended transmission line can be considered as a DUT. Transmission line has a ground pad beside the signal pad. For single ended transmission line characterization 2 ACP GS probes of $1000 \mu \mathrm{m}$ pitch are used. Before landing the ACP probes on the pads, it is well calibrated. It is also must to check probe alignment, probe planarization to achieve perfect contact. Measurement performed after successfully landing of probes on signal \& ground pads. With the help of this measured result RLGC parameter is calculated. Measured result is compared with simulated results \& other known solution (Port Extension).

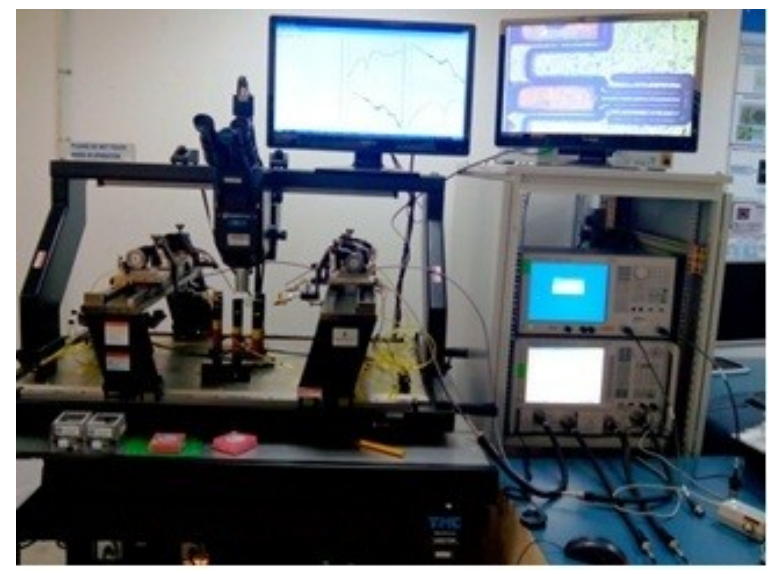

Figure 3: Measurement Setup

\section{Results}

S parameter data is measured using VNA. With the help of eq. (4) (5) (6) (7) RLGC parameter extracted \& it is shown in figure 4 below. Fig. 2 shows the S11, S21, S12, S22 measured by thefixture free characterization method. In fig.3, Insertion loss is compared with Port extension method.-3dB is achieved at 9 GHz. It shows that transmission line is capable of transmit information correctly till $9 \mathrm{GHz}$ after that loss occur. Measurement with Port Extension method introduces phase delay, it occurs due to inaccurate characterization of fixture \& its effects seen in RLGC parameter at high frequency. 


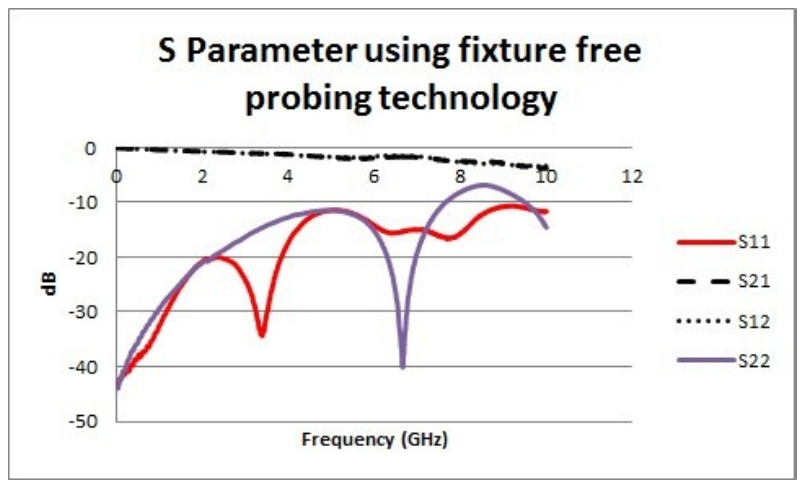

Figure 4: S Parameter using fixture free characterization method

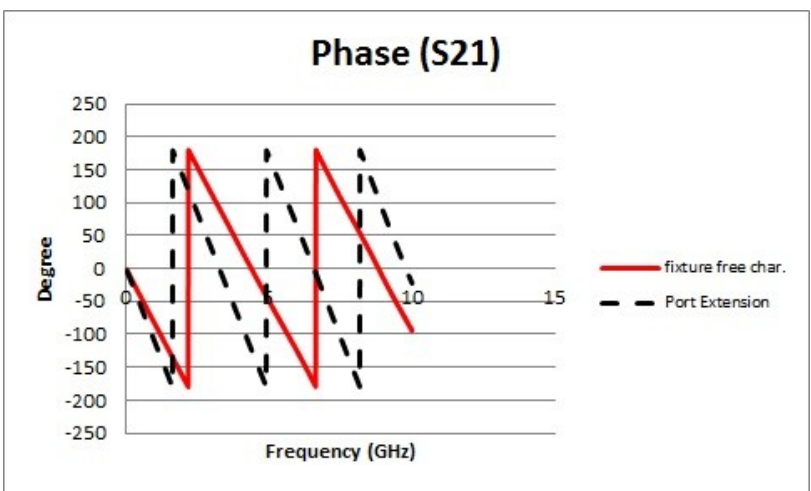

(A)

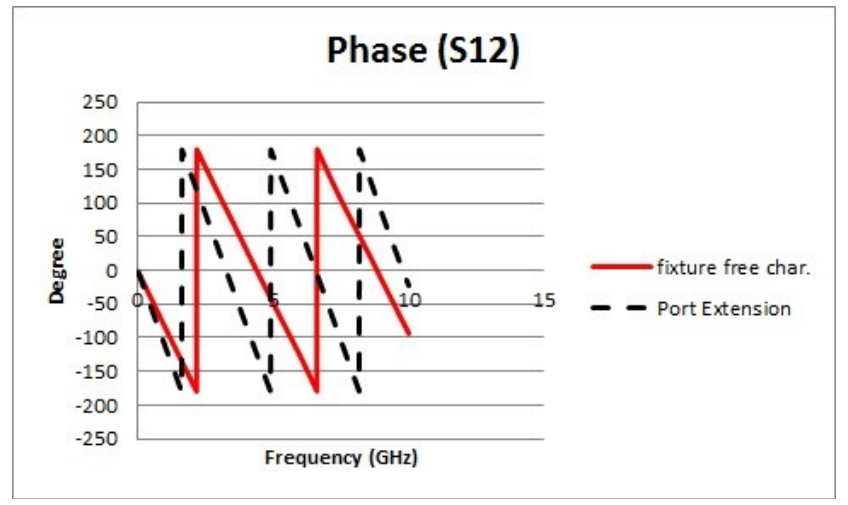

(B)

Figure 5: (A-B) Insertion Losses in Phase

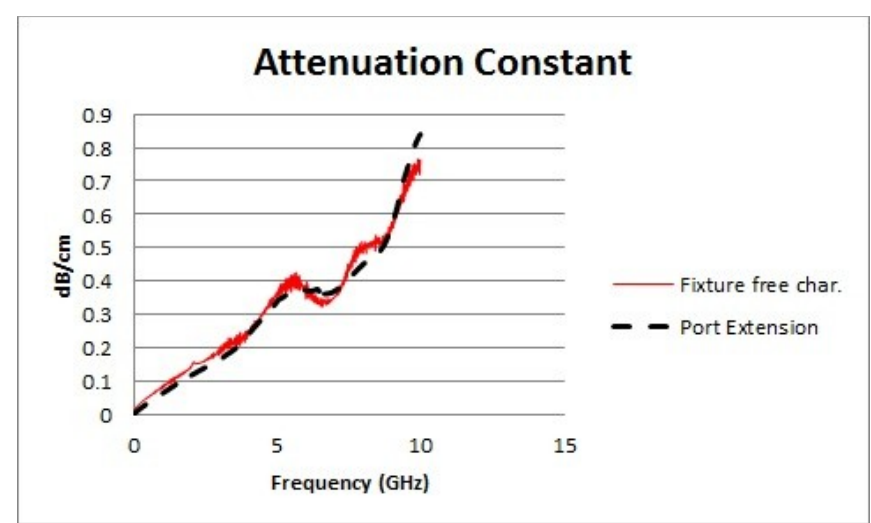

(C) 


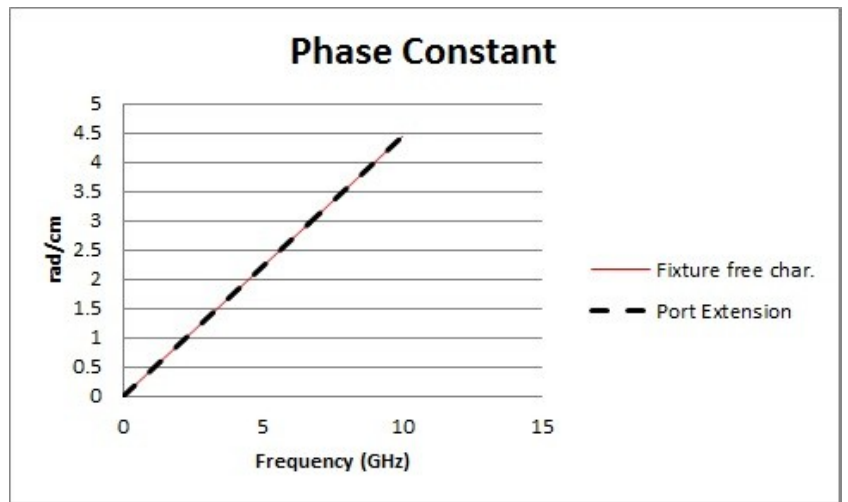

(D)

Figure 6: (E-F) Attenuation \& Phase Constant

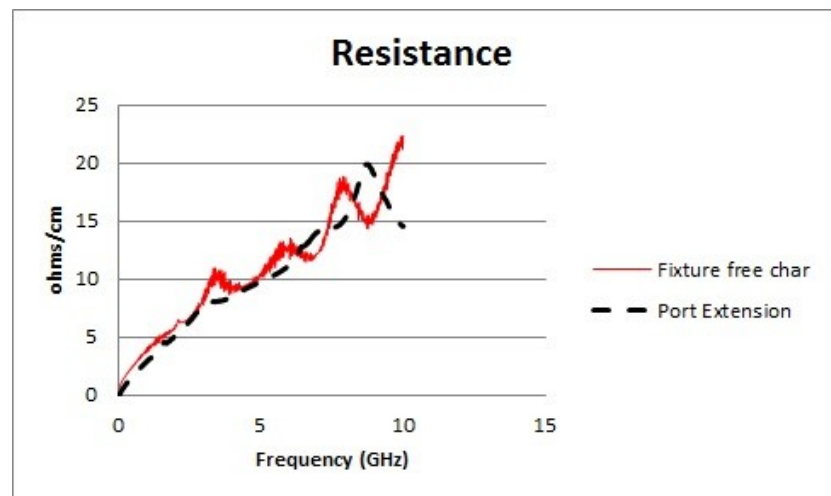

(E)

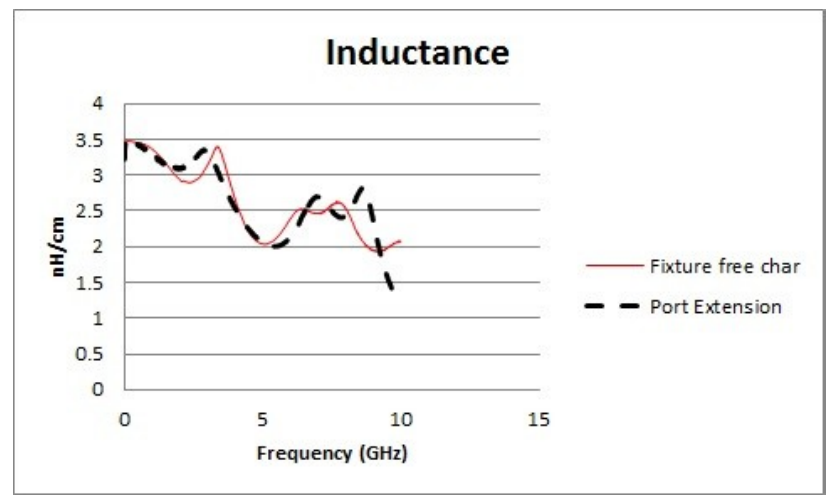

(F)

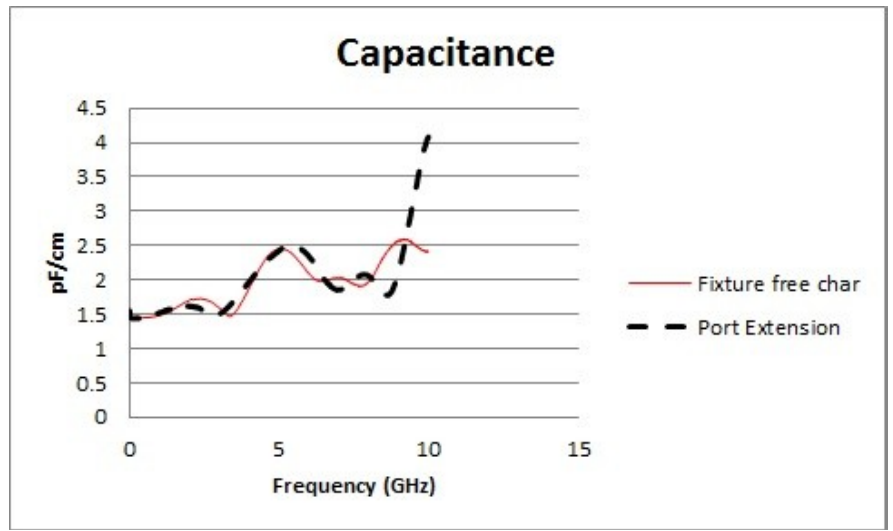

(G) 


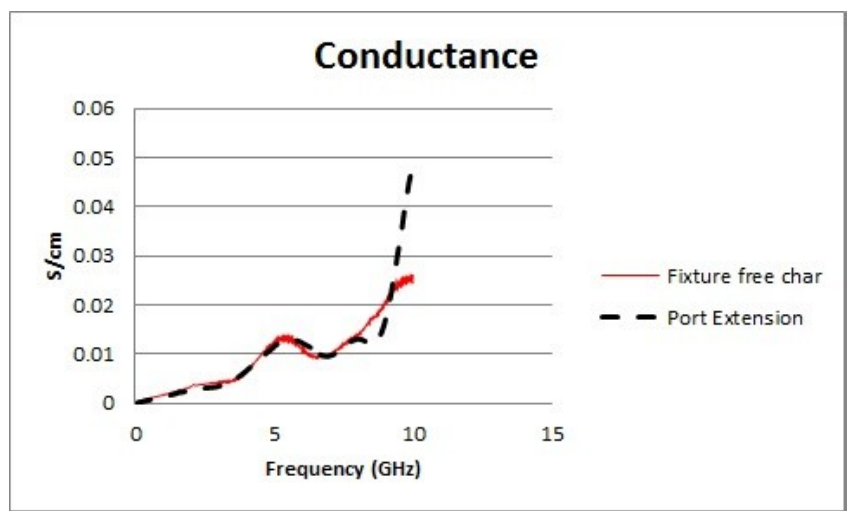

(H)

Figure 7: (E-H) RLGC Parameter

\section{Conclusion}

This paper shows a simple method for extraction of RLGC parameter of transmission line. Attenuation constant and phase constant is calculated using measured $\mathrm{S}$ parameter data. This method avoids complex calculation as used in other papers. This method is successfully applicable for lossy and lossless transmission line over a broad range of frequency. Fixture free measurement minimizes insertion \& return loss .Measurement setup is described \& results are mentioned.

\section{References}

[1]. Xiaoning Ye "De-embedding Errors due to Inaccurate Test Fixture characterization" IEEE Electromagnetic Compatibility Magazine-Volume 1-Quarter 4, 2012.

[2]. In-fixture measurements using vector network analyzers, Agilent Application Note AN1287-9.

[3]. Kazuki MAEDA, Kengo IOKIBE, Yoshitaka TOYOTA, Ryuji KOGA “ De-embedding of Board Parasitic with T-parameters for S-parameters of Integrated Circuits on PCB-Examinations in One-port Measurements-”.

[4]. Hongya $\mathrm{Xu}$, Erich Kasper “A De-embedding Procedure for One-port Active mm-Wave Devices” IEEE 2010.

[5]. Supreetha Rao Aroor, Rashaunda M. Henderson "Loss Performance of Planar Interconnects on FR-4 up to 67 GHz" IEEE Transactions on Components, Packaging and Manufacturing Technology. Volume 3, No. 12, December 2013.

[6]. Di Hu, Jaemin Shin, Timothy Michalka "Fixture-free Measurement Technique for PDN discrete Components" IEEE Electronic Components \& Technology Conference 2013.

[7]. Doug Campbell, Aldo Morales, Sedig Agili “An Improved Phase De-Embedding Technique for High Speed Connectors" IEEE 2010.

[8]. Reydezel Torres-Torres, Svetlana C "Extraction of the Model Parameters for the Attenuation in Printed Transmission Lines" IEEE Microwave and Wireless Components Letters, Vol.20, No. 12, December 2010.

[9]. Zhaoqing Chen, Sungjun Chun "Per-Unit-Length RLGC Extraction Using a Lumped Port De-Embedding Method for Application on Periodically Loaded Transmission Lines" IEEE Electronic Components \& Technology Conference 2006.

[10]. Guang Chen, Lin Zhu, Kathleen L. Melde "Extraction of Frequency Dependent RLGC Parameters of the Packaging Interconnects on Low-Loss Substrates from Frequency Domain Measurements" IEEE 2005.

[11]. Chuan-Lun Hsu, Gustavo Ardila, Philippe Benech "Parameters Extraction of Submicron Thin Film Microstrip Lines at Broadband mm-wave Frequency" IEEE Microwave Integrated Circuits Conference 2012. 